\title{
Use of Magnetic Resonance Tractography in Detecting Specific Features of the Impaiment of Conduction Pathways and Their Prognostic Value in Parkinson's Disease Complicated by Freezing of Gail
}

DOI: $10,17691 / \mathrm{stm} 2016.8 .4 .22$

Received May 16, 2016

A.G. Trufanov, MD, DSC, Lecturer, Department of Nervous Diseases

R.R. Khalimov, Head of Neurological Department';

A.A. Yurin, PhD Student, Department of Nervous Diseases';

1.V. Litvinenko, MD, DSc, Professor, Head of the Department of Nervous Diseases ${ }^{1}$

1S.M. Kirov Military Medical Academy, 6 Academic Lebedev St., Saint Petersburg, 194044, Russian Federation; 2354 District Military Clinical Hospital, 87 Dekabristov St., Ekaterinburg, 620144, Russian Federation

The aim of the study was to determine the localization of conductive pathways in patients with Parkinson's disease (PD) complicated with freezing of gait, by using data from magnetic resonance tractography, and to identify the prognostic value of these pathways in predicting the course of the disease.

Materials and Methods. 78 patients with idiopathic PD were examined and divided into two groups: patients with freezing of gait $(n=31)$ and without freezing of gait $(n=47)$. The protocol of the investigation consisted of clinical evaluation of the condition of the patients to determine the stage of the disease, and the use of a Siemens MRI Device (magnetic induction field, 1.5 T) to obtain standard images of the coronary, axial and sagittal planes, as well as MRI-protocols of the T1 gradient echo, plus diffusion-tensor tractography with further analysis of the findings with FreeSurfer software.

Results. The groups of patients showed statistically significant differences in the levels of the tract length, diffusivity and fractional anisotropy in the anterior thalamic radiations of both sides, the temporal area of the superior longitudinal fascicle on the left, the parietal area of the superior longitudinal fascicle on the right, and the corticospinal tract on the right. Analysis of the correlative tractographic values with the total scores on the walk-and-balance scale revealed that the highest prognostic value in the development of freezing of gait pertained to damage to the thalamo-frontal tract. A reduction in the value of the maximum voxel length to 80.0 or below, increased the risk of development of freezing of gait in 10 times.

Conclusion. The results demonstrate the involvement of conduction pathways in the mechanisms leading to freezing of gait in PD. Early diagnosis and prediction of the development of this complication using magnetic resonance tractography can indicate the need to adjust the medical treatment and to develop an appropriate special system of rehabilitation.

Key words: magnetic-resonance tractography; freezing of gait in Parkinson's disease; freezing of gait.

Introduction. Gait disorders in Parkinson's disease (PD) are some of the most significant motor disorders, their severity reflecting the severity of the patient's condition and their quality of life. Numerous recent studies have shown that gait disorders can be considered to be the fifth independent pivotal symptom of parkinsonism (along with hyperkinesia, rigidity, resting tremor and postural disorders), having their own pathogenetic mechanisms of development and requiring specific approaches to treatment [1-3].

As PD progresses, the typical gait pattern (a shuffling gait with shortened steps) changes and is accompanied by more complicated disorders manifested as impairment of walk initiation, instability when turning, freezing of gait
(FOG) and, as a result, by falls. Gait disorders in the late stages of PD stages can be attributed to freezing of gait that are integrative impairments of motion control that cannot be explained only by the damage to the brainstem and basal ganglia $[3,4]$. These disorders are caused by damage to the frontal lobes and their interconnections, as is indicated by the association of gait disorders in the late stages of PD with cognitive disorders reaching the level of dementia, predominantly of the frontal type. Disorders of gait control in the late stages of PD can be described as "primary", because they are caused by impairment of the process of choice in the initiation of locomotor and postural programs, rather than by their implementation, and because they do not depend on

For contacts: Artem G. Trufanov, e-mail: trufanovart@gmail.com 
any other neurological pathology (for example, sensitivity disorders, paresis or increased muscle tonus), nor are they changed by dopaminergic therapy or during the "on/ off" periods. Freezing of gait are particularly manifested in rough transition situations - at the start of walking; when turning; or when rising (i.e. when one motor program has to transition into another) and, therefore, they reflect a "defect in planning".

Many researchers [4-7] have evaluated cerebral anatomic structures that may be associated with freezing. We have identified pathological changes in the white matter in the auxiliary motor area, the premotor area, the orbitofrontal area, the caudate nucleus, the right parietal cortex, the putamen and the pedunculopontine nucleus.

Diffusion-tensor tractography (DTT) is a new method allowing us to visualize the fibers of the conduction pathways in vivo. In 2011 a group of Korean researchers [6] performed magnetic resonance tractographic investigations on 14 patients having PD complicated by freezing of gait in comparison with 24 patients without such disorders, by evaluating 7 areas of the brain. In these groups of patients they identified statistically significant differences in the level of fractional anisotropy in the pedunculopontine nucleus and the upper premotor areas on both sides, as well as in the right orbitofrontal cortex and the left auxiliary motor area, but they did not reveal any significant differences in the diffusivity levels. Furthermore, they did not assess the prognostic value of pathology in the white matter in relation to the freezing of gait in these patients.

The aim of the study was to determine the localization of lesions in the conductive pathways of patients with Parkinson's disease complicated by freezing of gait, by using data from magnetic resonance tractography, and to identify the prognostic value of the changes in these tracts in predicting the course of the disease.

Materials and Methods. We examined 78 right-handed patients with idiopathic PD diagnosed in accordance with the criteria of the British Brain Bank. All the patients presented with $3^{\text {rd }}$ stage PD according to the Hoehn and Yahr scale, 49 (63.6\%) with an akineticorigid form, and $29(36.4 \%)$ had a mixed form of PD. The patients were divided into two groups: with freezing $(n=31)$ and without freezing $(n=47)$. We used the Gait and Balance scale, GBS, which provides a more accurate measure of freezing than those of Section III of the Unified Parkinson's Disease Rating Scale or the FOG scale. Optimization of dopaminergic therapy in the groups of patients with freezing of gait did not lead to improvements in the gait nor to any reduction in the number of freezing episodes; neither did it show any connection with the development of freezing in the "off" periods. The general characteristics of the patients are presented in Table 1.

Table 2
Table 1

General characteristic of the patients in the study

\begin{tabular}{lccc}
\hline \multicolumn{1}{|c}{ Patients } & $\begin{array}{c}\text { Average age } \\
\text { (years) }\end{array}$ & $\begin{array}{c}\text { Duration } \\
\text { of the disease } \\
\text { (years) }\end{array}$ & $\begin{array}{c}\text { Score according } \\
\text { to the FOG scale } \\
\text { (points) }\end{array}$ \\
\hline With of gait $(n=31)$ & $62.7 \pm 5.4$ & $8.3 \pm 3.8$ & $30.7 \pm 10.2$ \\
\hline Without of gait $(n=47)$ & $61.1 \pm 6.2$ & $7.3 \pm 5.0$ & $8.0 \pm 3.9$ \\
\hline
\end{tabular}

All the patients underwent MRI on a Siemens (Germany) unit, using an induction magnetic field of 1.5 $\mathrm{T}$ to obtain standard images, T1-WI and T2-WI in the coronary, axial and sagittal planes. Furthermore a protocol of T1 gradient echo and DTT was used with all the patients, with subsequent analysis of the findings using FreeSurfer software. 16 anatomical conduction tracts from the right and left hemispheres of the brain were statistically analyzed. Correlations were calculated using Spearman's rho. The data were processed with Statistica 8.0 by StatSoft (Mann-Whitney test). To determine the odds ratio we used WinPepi for Windows software.

Results. We determined statistically significant differences between the groups of patients with PD in relation to the parameters of the main conduction pathways in the left (Table 2) and the right (Table 3) hemispheres of the brain. These differences concerned the tractographic characteristics of the cingulum bundle, the anterior thalamic radiations (thalamo-anterior tracts) and the temporal area of the longitudinal fascicles. We noted an increase in the values of radial diffusivity and a reduction in the fractional anisotropy along the line of the upper longitudinal fascicles in the temporal area, which testifies to its considerable involvement in the pathological process.

In the patients with freezing the right hemisphere had more severe damage of the white matter. The main component of the pyramidal system - the corticospinal tract - is involved in the pathological process, mainly through an increase in the medium diffusivity of the

\section{Differences between the parameters of the conduction pathways in the left hemisphere of patients with and without freezing (Me [LQ; UQ])}

\begin{tabular}{|c|c|c|c|}
\hline \multirow{2}{*}{ Localization of the tract } & \multicolumn{2}{|c|}{ Patients } & \multirow{2}{*}{$p$} \\
\hline & With freezing & Without freezing & \\
\hline Anterior thalamic radiation $\left(A D \cdot 10^{-6}\right)$ & $1,215[1,203 ; 1,277]$ & $1,185[1,172 ; 1,202]$ & 0.037 \\
\hline Cingulum bundle $\left(\mathrm{MD} \cdot 10^{-6}\right)$ & 825 [806; 832] & $779[764 ; 805]$ & 0.019 \\
\hline 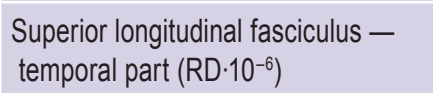 & $625[603 ; 734]$ & $588[559 ; 606]$ & 0.034 \\
\hline $\begin{array}{l}\text { Superior longitudinal fasciculus - } \\
\text { temporal part (FA) }\end{array}$ & $\begin{array}{c}0.4063 \\
{[0.3575 ; 0.4300]}\end{array}$ & $\begin{array}{c}0.4250 \\
{[0.3811 ; 0.4350]}\end{array}$ & 0.046 \\
\hline
\end{tabular}

$\mathrm{N}$ o t e s. MD: medium diffusivity, AD: axial diffusivity, RD: radial diffusivity, FA: fractional anisotropy. 
Table 3

Differences between the parameters of the conduction pathways in the right hemispheres of patients with and without freezing (Me [LQ; UQ])

\begin{tabular}{|c|c|c|c|}
\hline \multirow{2}{*}{ Localization of the tract } & \multicolumn{2}{|c|}{ Patients } & \multirow{2}{*}{$p$} \\
\hline & With freezing & Without freezing & \\
\hline Anterior thalamic radiation (MaxL) & $80.0[74.0 ; 84.0]$ & $98.0[83.0 ; 102.0]$ & 0.021 \\
\hline Corticospinal tract $\left(\mathrm{RD} \cdot 10^{-6}\right)$ & $630[616 ; 668]$ & $555[521 ; 564]$ & 0.030 \\
\hline Corticospinal tract $\left(\mathrm{MD} \cdot 10^{-6}\right)$ & $870[837 ; 891]$ & $787[762 ; 810]$ & 0.028 \\
\hline Inferior longitudinal fasciculus (AvL) & $73.0[72.0 ; 75.0]$ & $85.0[81.0 ; 87.0]$ & 0.015 \\
\hline $\begin{array}{l}\text { Superior longitudinal fasciculus - } \\
\text { temporal part }\left(\mathrm{RD} \cdot 10^{-6}\right)\end{array}$ & $640[614 ; 691]$ & $592[566 ; 611]$ & 0.011 \\
\hline $\begin{array}{l}\text { Superior longitudinal fasciculus - } \\
\text { temporal part (MD·10-6) }\end{array}$ & $827[791 ; 852]$ & $767[759 ; 779]$ & 0.032 \\
\hline
\end{tabular}

Notes. MaxL: maximum voxel length, AvL: average voxel length, MD: medium diffusivity, RD: radial diffusivity.

conductor. As in the dominant hemisphere, the right hemisphere has damaged upper longitudinal fascicles, however there is also damage not only in the temporal, but additionally in the parietal area.

As well as differences in the upper longitudinal fascicles, these groups of patients had statistically significant differences in the characteristics of the lower longitudinal fascicles - the links between the occipital and temporal lobes. When there is freezing, the anterior thalamic radiation has a bilateral nature, and in the right hemisphere there is a reduction in the length of this tract.

The localization and the courses of the conduction pathways most involved in the pathological process of the patients with freezing are represented in Figures 1-6.

To identify possible markers of the development of freezing of gait in PD we performed a correlation analysis between the tractographic parameters and the GBS scores using Spearman's rho (Table 4).

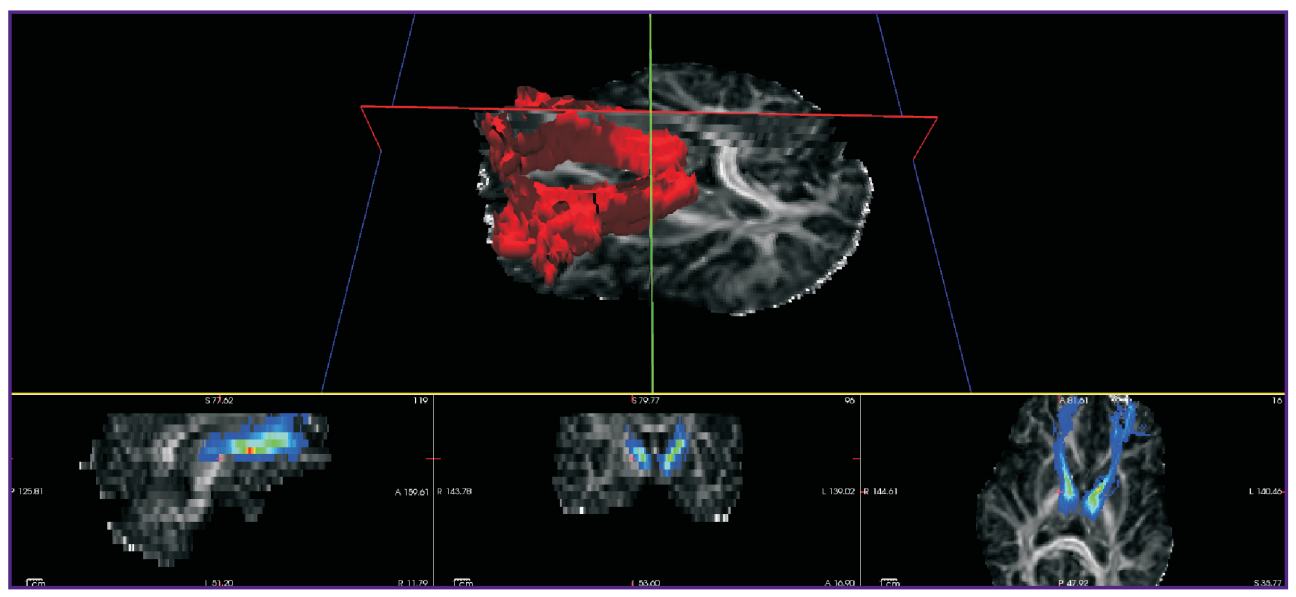

Figure 1. Localization and the course of the thalamo-frontal tract

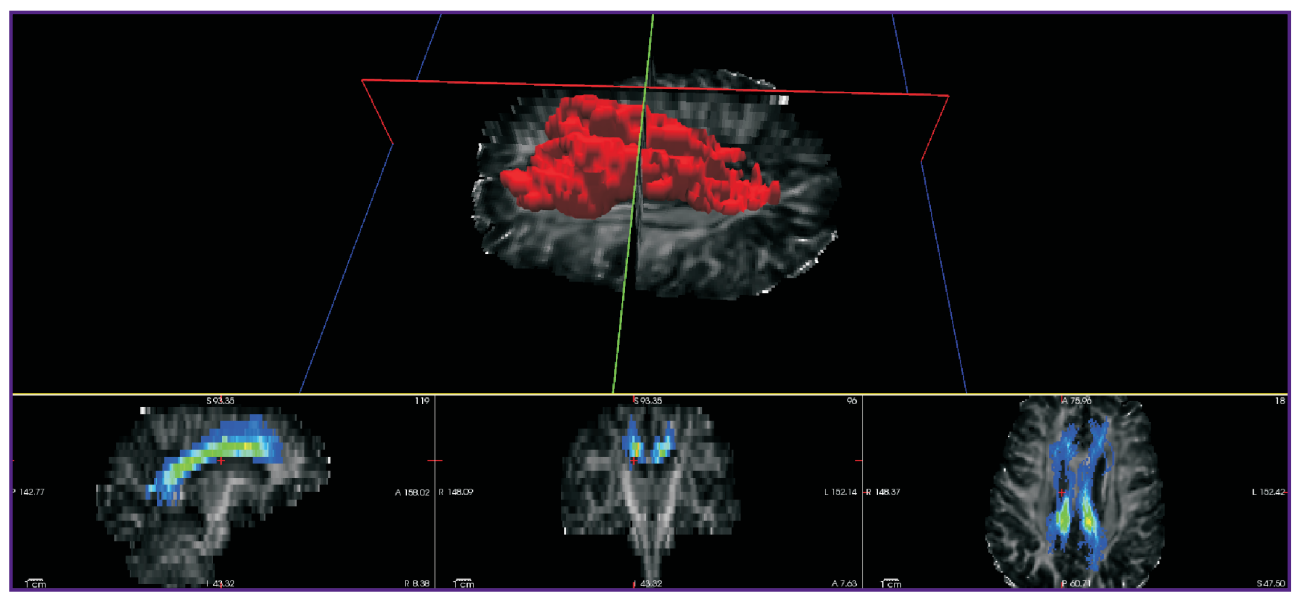

Figure 2. Localization and the course of the cingulum bundle 


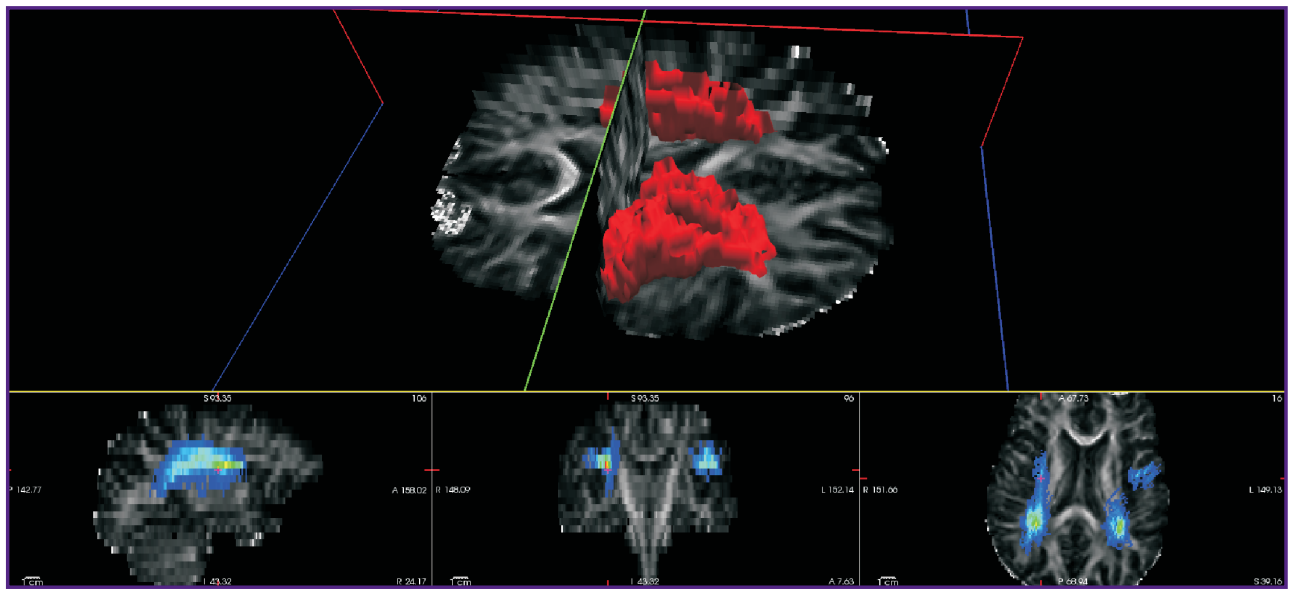

Figure 3. Localization and the course of the superior longitudinal fasciculus in the temporal area

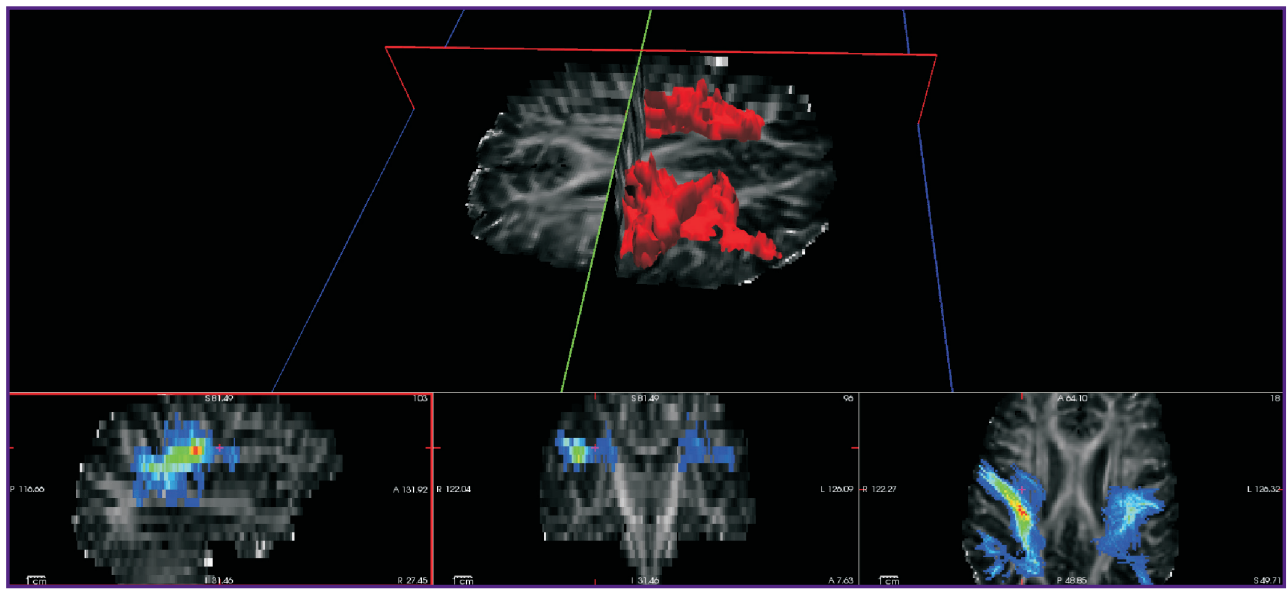

Figure 4. Localization and the course of the superior longitudinal fasciculus in the parietal area

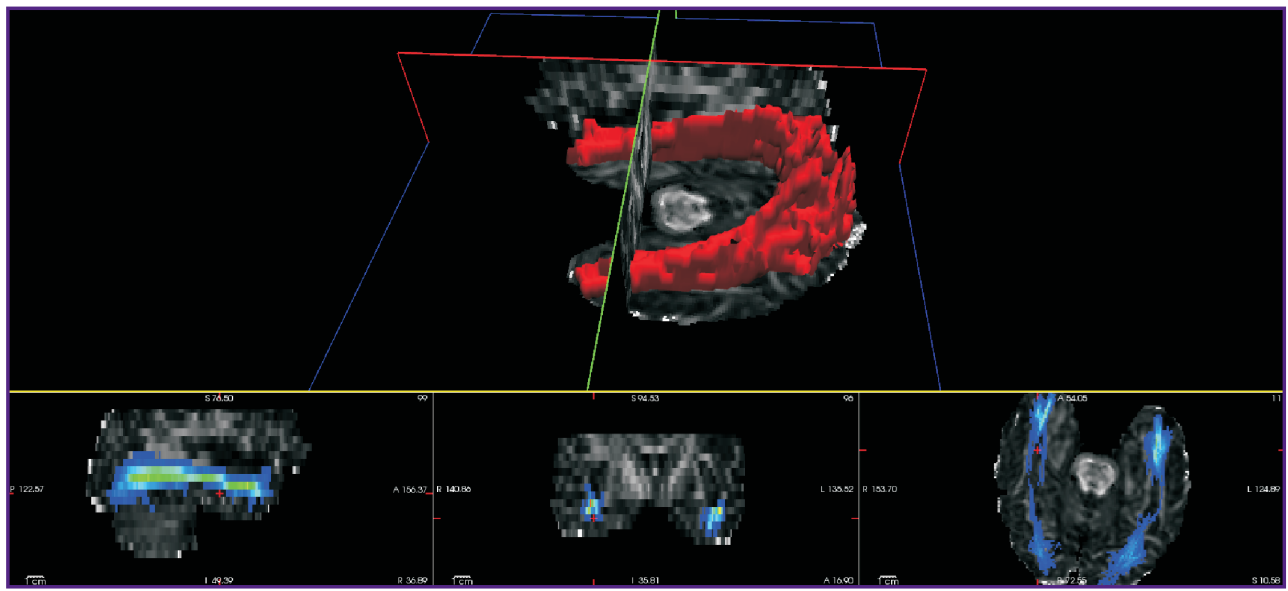

Figure 5. Localization and the course of the inferior longitudinal fasciculus

When the dominant hemisphere was evaluated, freezing had a strong positive correlation only with radial diffusivity in the angular bundle that was part of the limbic system, providing frontal-temporal interactions.
Evaluation of the right hemisphere indicated that freezing had a strong negative correlation with the level of fractional anisotropy of the lower longitudinal fascicles, as well as indicating explicit positive links 


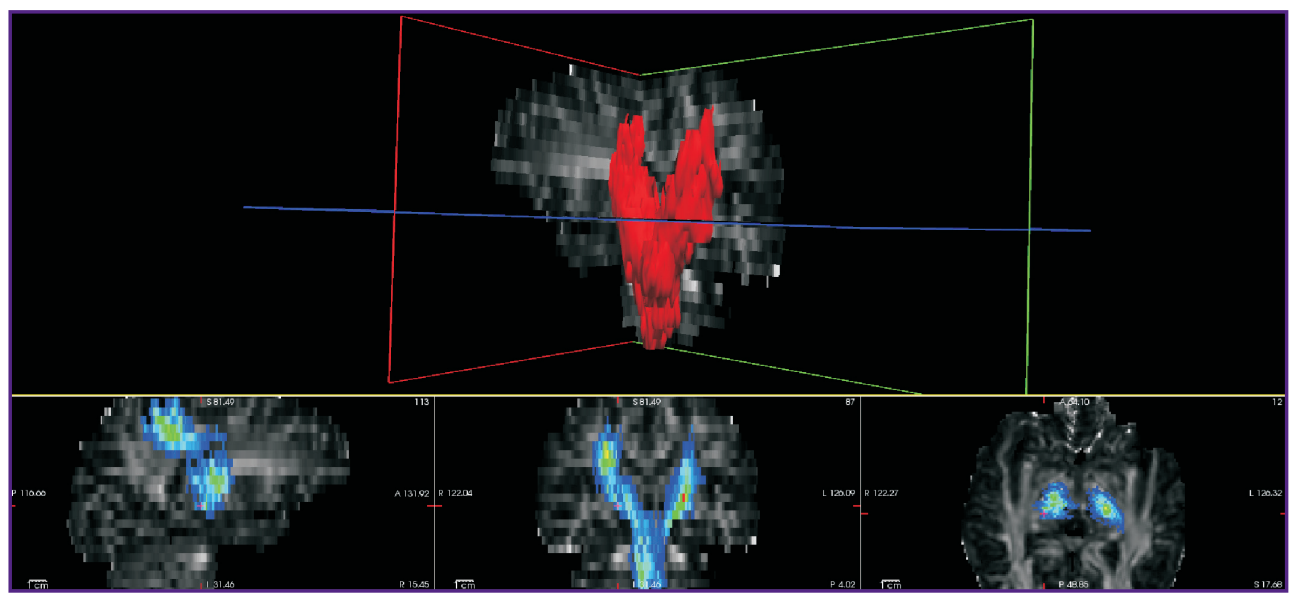

Figure 6. Localization and the course of the corticospinal tract

Table 4

Correlation of magnetic resonance tractography with the GBS scores in patients with freezing $(p<0.05)$

\begin{tabular}{ll}
\hline \multicolumn{1}{c}{ Localization } & \multicolumn{1}{c}{ Seft hemisphere of the brain } \\
\hline \multicolumn{2}{|c|}{ Right hemisphere of the brain } \\
\hline Angular part of cingulum bundle (RD) \\
\hline \multicolumn{2}{|c|}{0.74} \\
\hline Anterior thalamic radiation (RD) & 0.89 \\
\hline Anterior thalamic radiation (MD) & 0.83 \\
\hline Anterior thalamic radiation (FA) & -0.85 \\
\hline Inferior longitudinal fasciculus & -0.91 \\
\hline
\end{tabular}

N o t e s. MD: medium diffusivity, RD: radial diffusivity, FA: fractional anisotropy.

between the freezing phenomenon and damage to the thalamo-frontal tract.

To determine the risk of freezing depending on the values of the tractographic parameters we calculated the odds ratio for the right anterior thalamic radiations (OR10.0). As the critical values we used the medians ( $\mathrm{Me}-$ 80.0 ) and $95 \%$ confidence intervals (2.41-20.30) of the corresponding parameters in the patients with freezing of gait $(p<0.001)$.

Discussion. The findings suggest that the effects of extensive damage to the white matter of the brain in PD are complicated by freezing of gait. It is possible to identify certain tracts which have the biggest impact on the development of this complication, and therefore have prognostic value.

In our opinion the most important tracts of all those investigated is the anterior thalamic radiation and the thalamo-anterior path. It is known that thalamo-frontal path is an integral part of the cholinergic motion control system. For this conductor we detected not only multiple differences between the groups of patients with freezing and without freezing, but also strong correlations of the corresponding parameters with the walk-and-balance scale. The statistically significant negative relationships between postural disorders and the parameters of fractional anisotropy are worth mentioning. If a focus on diffusivity parameters is difficult due to the limited range of values, a periodical analysis of fractional anisotropy can be quite valuable from the prognostic point of view. If the patient has a dynamic reduction in the fractional anisotropy of the thalamo-frontal path, one can expect the development of freezing, caused mainly by the dysfunction of the frontal lobes. Such a prognosis allows the prescribing of an effective therapy as soon as possible.

According to our data damage in the upper longitudinal fascicles is a particularly important indicator of the development of gait disorders. It was found that the temporal area of the right longitudinal fascicle suffers mainly in the left (dominant) hemisphere whereas it is the parietal part that is mainly involved in the case of the right hemisphere. Taking into account the considerable spread of this conductor and the presence (except in the frontal, parietal and temporal areas) of a large number of sub-bundles providing information exchange between the most significant integrative fields of the lobes of the hemisphere, dysfunction of the upper longitudinal fascicles can, to a great extent, determine the pathogenesis of freezing of gait. The developing dysfunction of this conductor is a secondary effect and is the result of considerable damage within the brain cortex at late stages of PD [8, 9].

This paper has shown the increase in the radial and medium diffusivity of the right corticospinal pathways in the case of gait disorders. Further studies of the damage to these structures are of interest, because some PD patients also complain of reduced strength in their limbs. These complaints appear mainly subjective, given that dynamometry, as a rule, does not show any changes in muscular strength. It should be noted that the identified pathology of the corticospinal pathways and of the precentral fissures does not lead to the formation of an 
actual pyramidal insufficiency, excepting the presence of pathological reflexes in these patients [10,11].

In patients with PD and gait disorders, taking into account the signs of the involvement of the thalamofrontal tract in the pathological process, it would be promising to study, further, the effectiveness of a combined approach to the correction of motor disorders usingcholinergic medications and repeated sessions of transcranial magnetic stimulation [12].

Conclusion. Early diagnosis and forecasting of freezing in PD using magnetic-resonance tractography can help in adjusting medical therapy and in the development of bespoke systems of rehabilitation.

Study Funding and Conflicts of Interest. This study was not supported by any financial sources and there is no topic specific conflicts of interest related to the authors of this study.

\section{References}

1. Damulin I.V., Pavlova A.I. Dementia of frontal type. Nevrologicheskiy zhurnal 1997; 1: 37-42.

2. Damulin I.V., Zhuchenko T.D., Levin O.S. Narushenie ravnovesiya i pokhodki u pozhilykh. $\mathrm{V}$ kn.: Dostizheniya $v$ neyrogeriatrii. T. 1 [Balance and gait disorders in elderly people. In: Achievements in neurogeriatrics. Vol. 1] Pod red. Yakhno N.N., Damulina I.V. [Yakhno N.N., Damulin I.V. (editors)]. Moscow; 1995; p. 71-97.

3. Levin O.S. Narusheniya khod'by: mekhanizmy, klassifikatsiya, printsipy diagnostiki i lecheniya. V kn.: Ekstrapiramidnye rasstroystva [Gait disorders: mechanisms, classification, and principles of diagnosis and treatment. In: Extrapyramidal disorders]. Pod red. Shtoka V.N., IvanovoySmolenskoy I.A., Levina O.S. [Shtoka V.N., IvanovaSmolenskaya I.A., Levin O.S. (editors)]. Moscow; 2002; p. 473-494.

4. Levin O.S., Yunishchenko N.A., Skvortsov D.V. Klinikoinstrumental'nyy analiz khod'by i postural'noy neustoychivosti pri bolezni Parkinsona. V kn.: Materialy VII Mezhdunarodnoy konferentsii "Sovremennye tekhnologii vosstanovitel'noy meditsiny" [Clinical-instrumental analysis of gait and postural instability in Parkinson's disease. In: Materials of the VII International Conference "Modern technology in rehabilitation medicine"]. Sochi; 2004; p. 15-18.

5. Bartels A.L., Balash Y., Gurevich T., Schaafsma J.D., Hausdorff J.M., Giladi N. Relationship between freezing of gait (FOG) and other features of Parkinson's: FOG is not correlated with bradykinesia. J Clin Neurosci 2003; 10(5): 584-588, https://doi.org/10.1016/S0967-5868(03)00192-9

6. Grossi D., Trojano L., Pellecchia M.T., Amboni M., Fragassi N.A., Barone P. Frontal dysfunction contributes to the genesis of hallucinations in non-demented Parkinsonian patients. Int J Geriatr Psychiatry 2005; 20(7): 668-673, https:// doi.org/10.1002/gps.1339.

7. Ito K., Nagano-Saito A., Kato T., Arahata Y., Nakamura A., Kawasumi Y., Hatano K., Abe Y., Yamada T., Kachi T., Brooks D.J. Striatal and extrastriatal dysfunction in Parkinson's disease with dementia: a 6-[18F]fluoro-L-dopa PET study. Brain 2002; 125 (Pt 6): 1358-1365, https://doi. org/10.1093/brain/awf134.

8. Trufanov A.G., Odinak M.M., Litvinenko I.V., Rezvantsev M.V., Voronkov L.V. Early diagnosis of dementia with the help of MR-morphometry in patients with Parkinson's disease. Voenno-meditsinskiy zhurnal 2013; 334(9): 29-34.

9. Litvinenko I.V. Dementia and psychotic disorders in parkinsonism: common origin and new perspectives in therapy. Uspekhi gerontologii 2004; 13: 94-101.

10. Litvinenko I.V., Mikhailenko A.A., Trufanov A.G., Anoshina E.A., llyinskiy N.S. Neuroimaging basement of pathological reflexes in 2-3 stages (Hoehn, Yahr scale) of Parkinson disease. Vestnik Rossiyskoy voenno-meditsinskoy akademii 2012; 4(40): 116-120.

11. Trufanov A.G., Litvinenko I.V., Efimtsev A.Yu. The anatomical brain pathways affection in advanced 2-3 stages (Hoehn, Yahr scale) of Parkinson disease. Vestnik Rossiyskoy voenno-meditsinskoy akademii 2012; 4(40): 36-41.

12. Litvinenko I.V., Khalimov R.R., Trufanov A.G., Krasakov I.V., Haimov D.A. New approach to gait disorders therapy in late stages of Parkinson's disease. Uspekhi gerontologii 2012; 25(2): 267-274. 